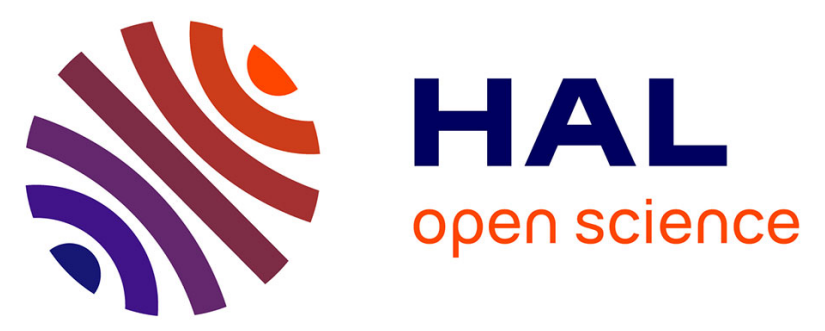

\title{
Reasoning on the Risks of Dynamic Manufacturing Networks through Cognitive Mapping
}

Ourania Markaki, Sotirios Koussouris, Panagiotis Kokkinakos, Dimitris

Panopoulos, Dimitris Askounis

\section{To cite this version:}

Ourania Markaki, Sotirios Koussouris, Panagiotis Kokkinakos, Dimitris Panopoulos, Dimitris Askounis. Reasoning on the Risks of Dynamic Manufacturing Networks through Cognitive Mapping. 15th Working Conference on Virtual Enterprises (PROVE), Oct 2014, Amsterdam, Netherlands. pp.589596, 10.1007/978-3-662-44745-1_58 . hal-01392164

\section{HAL Id: hal-01392164 \\ https://inria.hal.science/hal-01392164}

Submitted on 4 Nov 2016

HAL is a multi-disciplinary open access archive for the deposit and dissemination of scientific research documents, whether they are published or not. The documents may come from teaching and research institutions in France or abroad, or from public or private research centers.
L'archive ouverte pluridisciplinaire HAL, est destinée au dépôt et à la diffusion de documents scientifiques de niveau recherche, publiés ou non, émanant des établissements d'enseignement et de recherche français ou étrangers, des laboratoires publics ou privés.

\section{(c)(1)}

Distributed under a Creative Commons Attribution| 4.0 International License 


\title{
Reasoning on the Risks of Dynamic Manufacturing Networks through Cognitive Mapping
}

\author{
Ourania Markaki ${ }^{1}$, Sotirios Koussouris ${ }^{1}$, Panagiotis Kokkinakos ${ }^{1}$, \\ Dimitris Panopoulos ${ }^{1}$ and Dimitris Askounis ${ }^{1}$ \\ ${ }^{1}$ Greek Interoperability Centre, Decision Support Systems Lab, School of Electrical and \\ Computer Engineering, National Technical University of Athens, \\ Iroon Polytechniou str. 9, 15780 Zografou, Athens, Greece \\ \{omarkaki, skous, pkokkinakos, dpano, askous\}@epu.ntua.gr
}

\begin{abstract}
Dynamic Manufacturing Networks (DMNs) are increasingly deemed to be the evolution of typical supply chains in the manufacturing sector and a promising solution in the light of the enterprises' effort to remain flexible and competitive in today's rapid changing and demanding environment. The decision of joining a DMN however is quite important for any enterprise, since beside significant benefits it brings about changes in the way it operates, bearing thus also considerable risks. This paper attempts to cultivate an understanding around the risks of DMN participation, utilizing the causal characteristics of Cognitive Maps (CMs) for identifying the causes of unsatisfactory or unaccepted DMN operation outcomes. It further elaborates on how DMN risks are inherently dealt with through the IMAGINE framework that guarantees business alignment and interoperability.
\end{abstract}

Keywords: Dynamic Manufacturing Networks, DMNs, Risks, Cognitive Maps, CMs, Interoperability, Causal Relationships.

\section{Introduction}

With increasing competition in the global market, manufacturing enterprises are seeking ways to improve their core capabilities, streamline their processes, establish strategic alliances, and respond in a more agile way than in the past so as to leverage spontaneous business opportunities, and thereby position themselves for survival in the future [1]. One of the most promising solutions in their pursuit seems to be the concept of "upgrading" their supply chains to Dynamic Manufacturing Networks (DMNs). The DMN concept evolves and particularizes the notion of virtual enterprises [2] in the manufacturing sector, as it is a dynamic alliance of manufacturing entities collaborating for gaining mutual benefits [3-5]. Compared to a virtual enterprise however, a DMN is a real formation, though with loose ends and a quite flexible structure, which includes geographically dispersed OEMs and a pool of potential suppliers of various tiers.

For an enterprise, participating in (or forming) a DMN can be considered as a systematic way for cultivating extended co-operation with other members of its 
supply chain, and calls for modifications in its modus operandi, while it necessitates the network's dynamic management, so as to enable business alignment and active collaboration among the different network nodes [6]. In this respect, it also creates the need for stimulating and supporting communication concerning the impact of change, and thereby concerning the risks involved, which have to be identified and assessed [7], as the tighter relationships mean more dependencies between the companies.

This paper proposes an approach for reasoning on the risks of participating in a DMN, based on Cognitive Maps (CMs), i.e. mathematical models that hold the advantage of portraying information about a system more succinctly than the corresponding textual description [8], and suggest themselves as a means of bringing forth the way of thinking and making visible the perceptions of individuals. The paper leverages the causal characteristics of CMs as a modelling technique for generating a network of interdependent DMN risk-related factors and identifying the causes of DMN failure.

The proposed approach has both theoretical and practical benefits. Given the novelty and controversy around the concept of DMNs, such a succinct mechanism of conveying the dynamics of this new modus operandi and its associated risks is believed to be useful for any enterprise contemplating or undertaking major organizational changes towards its involvement in a DMN. Thus, the proposed model targets primarily DMN managers, high level executives, BPR consultants etc., assisting them to reason effectively on the risks involved in such formations and to devise appropriate mitigation strategies; nevertheless the explanatory nature of the model can also prove to be useful in a wider educational setting.

This paper consists of five sections. Section 2 introduces the novel and innovative concept of DMNs as well as the modelling instrument of CMs. Section 3 focuses on the application of the latter with the aim of stimulating reasoning on the risks involved in DMNs. Section 4 particularizes the identified concepts and developed CM in the case of IMAGINE, highlighting the paramount importance of interoperability. Finally, Section 5 summarizes the ideas presented and draws relevant conclusions.

\section{Background}

\subsection{Dynamic Manufacturing Networks}

A DMN is a coalition, either permanent or temporal, comprising production systems of geographically dispersed Small and Medium Enterprises (SMEs) and/or Original Equipment Manufacturers (OEM) that collaborate in a shared value-chain to conduct joint manufacturing [3]. Each member of the network produces one or more product components that can be assembled into final service-enhanced products under the control of a joint production schedule. Production schedules are monitored collectively to accomplish a shared manufacturing goal, while products are composed, (re-) configured and transformed on demand through dynamic and usually ad-hoc inter-organizational collaborations that can cope with evolving requirements.

The decision of joining a DMN is, as already discussed, quite an important one for any enterprise, since it is related to many changes not only to the way the enterprise 
collaborates with the external environment, but also to the way that almost all the internal processes are being performed; bearing thus also considerable risks. This is why it is important to cultivate an understanding of how the enterprise's participation in a DMN generates risks for the operation of the latter. To this end a closer look on the way in which a DMN is structured is required.

The DMN lifecycle, developed within the context of the IMAGINE FP7 FoF project [3], [9] is an innovative approach for modelling the entire lifespan of a DMN, ranging from planning and sourcing, to manufacturing and delivery. The DMN lifecycle places structure on the temporal order of the activities required for setting up and running a Dynamic Manufacturing Network, thereby encompassing the phases of i.) Network Analysis and Configuration, ii.) Network Design, and iii.) Network Execution, Management and Monitoring. The lifecycle is in fact initiated by a customer request that outlines the types and numbers of products to be produced, as well as the timeframe in which the product(s) should be ready. The Network Analysis and Configuration phase includes then the engagement of manufacturing partners, resources and other product-related information into a joint manufacturing schedule. The Network Design phase lies in combining all crucial manufacturing and physical processes, e.g., machining, forging, casting, and injection moulding in an end-to-end fashion, producing thus a chart of workflows that constitutes a working canvas for the entire DMN. Finally, the Network Execution, Management and Monitoring phase involves the actual deployment of the network and its processes and all the necessary activities for monitoring and troubleshooting its operation.

\subsection{Cognitive Maps}

Cognitive maps were first introduced by political scientist Robert Axelrod [10] in the 1970 s as a means of representing social scientific knowledge. A CM is a network diagram depicting causes and effects [11], and is represented by a labelled, directed graph of nodes and edges. Nodes represent domain concepts and edges causal relationships between the nodes. The direction of an edge reveals the direction of a causal relationship, which is also called a feedback. A positive (negative) feedback from node $\mathrm{A}$ to node $\mathrm{B}$ means that an increase in variable A causally increases (decreases) variable B.

CMs are acknowledged as cognitive due to the fact that they use concepts to elicit and represent perceptions. They have been used in a variety of contexts, most prominently in operations management [12] as a means of facilitating brainstorming and communication. Thanks to their characteristics, CMs suggest themselves as a suitable method for capturing the complex interactions among concept variables in the DMN environment.

\section{Causal Mapping of Risk Factors in DMNs}

The proposed framework for modelling DMN risks through CMs has been developed as the result of a benefits and risks study carried out within the IMAGINE project [3], 
as a means of identifying business drivers for attracting enterprises into joining DMNs. Data gathering was done through semi-structured interviews with industry stakeholders and information collected was analyzed using a grounded theory approach [13] that served to qualitatively identify all factors putting at stake the operation of both the single enterprise and the network as a whole. It is noted that the factors identified in this paper as well as their correlations are generic enough and can be applied to any DMN-related scenario. The list of concept variables elicited by the stakeholders' observations includes the following factors, provided thereafter along with a short explanation:

Lack of Appropriate Information, indicating incomplete or invalid information sharing as well as insufficiency of information exchange within the DMN network.

Unsuitable Partner Selection, denoting low compatibility and appropriateness of long/short listed, and thereby finally selected partners in terms of their capabilities, financial bid and quality offered.

Poor Network Configuration, referring to a low level of aptness of the baseline network configuration, including the preliminary, high-level production schedule.

Poor Network Design, indicating accordingly poor adequacy of the network design, including the final production schedule.

Poor Network Execution, Monitoring \& Management, corresponding to ineffective handling and troubleshooting of the network operation.

Unwanted and Malicious Attacks, pertaining to the event of a third party gaining, usually through malicious software, unauthorized access to private computer systems or sensitive information, causing thus disruptions in the DMN information systems' operation.

Information Leaks, denoting the occasion at which some information, either sensitive or not, is revealed to unauthorized parties.

Strategic or $R \& D$ Knowledge Misuse, relating to the event at which some information leakage results also in important knowledge misuse by former DMN members or DMN competitors.

Disclosure of Unnecessary Data, referring to the unreasonable exposure of nonessential for the manufacturing course, but possibly sensitive information.

Project Failure, relating to the unfortunate event that the DMN fails to meet a particular project's requirements, the latter pertaining to time, quality or cost constraints.

DMN Dissolution, denoting the termination of the DMN operation mostly as a result of a key partner's withdrawal or the failure of a project undertaken.

Partner Withdrawal, pointing to the event that a DMN member drops out of the network and has thus to be replaced.

Key Partner/Supplier Withdrawal, identified as the above risk, however bearing more serious impacts, since the enterprise that drops out of the network is a key DMN member.

Partner Shortcoming, indicating situations in which a DMN member fails to meet the time, cost or quality constraints and specifications ascribed to it in the context of the manufacturing process.

Damage to DMN Reputation, mapping to the risk of the DMN reputation being negatively affected as a result of the individual partners' and whole network's inefficient performance. 
High Integration Cost, referring to the investments required for individual partners to make their IT systems compliant with the DMN platform and engage in the DMN platform functionality.

Financial Exposure of Partners, pertaining to the financial risk undertaken by DMN partners due to their participation in the network.

Production Delays/Mistakes, indicating situations at which design or production flaws decelerate or retrograde the manufacturing process.

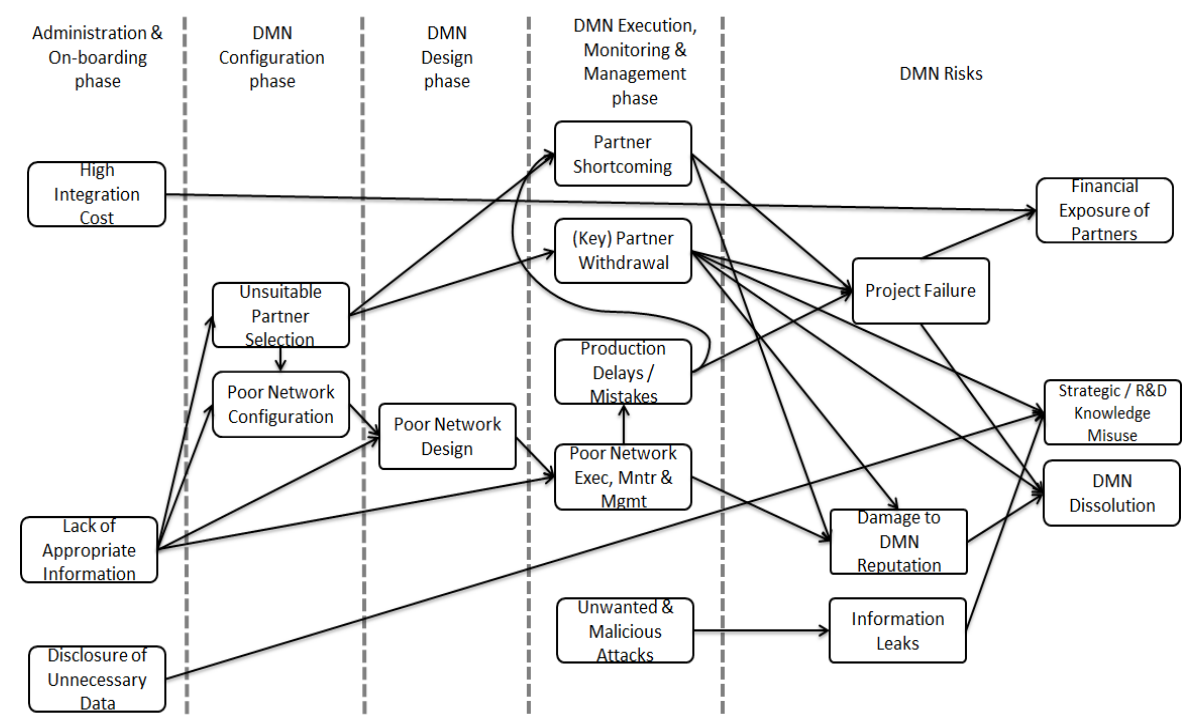

Fig. 1. Cognitive Map of concept variables relating to DMN risks

What is noticeable with this list is that some concept variables clearly constitute risk outcomes, i.e. effects or consequences of preceding problems, as in the case of "Project Failure" or "DMN Dissolution", while others even though not pointing directly to an unfortunate event, and being less critical, might contribute to the occurrence of such, in conjunction with other factors, thus being identified as riskrelated factors. Additionally, some of the concept variables identified seem to be present in more than one DMN phases, while others are specific to a particular stage. Thereby, in modelling DMN risk-related factors, it has been found helpful to take into account the structure of the DMN lifecycle and map the former according to their occurrence within the physical stages of the latter. In view of facilitating this task, in addition to the three lifecycle phases described in Section 2.2, a fourth one, that of Administration and On-Boarding has been added, covering all the preparatory steps preceding the emergence of a request for manufacturing a product, i.e. the activities related to bringing together the DMN actors in the context of a vertical marketplace and making the necessary technical and organizational arrangements to ensure technical connectivity, access to the appropriate information etc. 
What is required as a next step is the identification of cause and effect relationships between the risk-related factors and the actual risk outcomes. Below, the authors present some of the highlights of the raw correlations brought up by the stakeholders interviewed:

- An unsuitable selection of partners increases the chances of a partner not being able to perform as planned, which in turn amplifies the risk of a project failure.

- Exchange of invalid partner information across the network undermines the efficiency and effectiveness of network monitoring and management, thus possibly causing production delays and mistakes.

- A key partner's withdrawal holds the potential to damage the network's reputation or even cause the DMN dissolution, while in parallel raising the likelihood of project failure or knowledge misuse afterwards.

- Strategic or R\&D knowledge misuse constitutes an important risk, which is additionally influenced by the disclosure of unnecessary data and the failure to prevent information leakages in general.

- The financial exposure of each partner is highly affected by the initial onboarding cost as well as by the actual outcome of the DMN operation, i.e. the success or failure of the project undertaken.

Looking at the above list of correlations, one may notice that a risk outcome may be linked to several risk-related factors. For instance, a project failure may occur as a result of a partner shortcoming or withdrawal or a production delay/mistake not dealt with efficiently and timely, or even a combination of these factors. In other cases, the risk probability generated by a concept variable becomes a factor that leads to some other risk outcome. For example, the possibility of information leaks grows with the number of unwanted and malicious attacks, while in parallel raising the risk of strategic or R\&D knowledge misuse.

The relationships between the factors possibly leading to unaccepted or unsatisfactory DMN operation outcomes seem therefore to be more complex than what the above simple list of cause and effect relationships implies. In this context, the authors leverage the instrument of CMs to create a richer picture on the risks involved in the novel and promising approach of DMNs, and thereby on the respective mitigation strategies required. The full set of cause and effect relationships among the identified DMN risk-related factors are presented in Fig. 1.

\section{Reasoning on the DMN Risks: the IMAGINE case}

With the view of applying the CM of Section 3 in the case of the IMAGINE project, which actually targets the development and delivery of a novel methodology and the respective platform for effective end-to-end management of DMNs in a plug and produce approach, one has to highlight that the cornerstone of the latter is the DMN Blueprint Model. The former is a declarative meta-model that aggregates and modularizes production, manufacturing operations management and logistics information by specifying four types of inter-related blueprints [9], as follows: 
The Partner Blueprint (Partner BP), which provides both static and dynamic, business and technical information to facilitate partners' selection by a specific contractor;

the Product Blueprint (Product BP), containing all components required, i.e. machines, tools, personnel skills, materials, other equipment, for producing a product, as well as other entities necessary for the manufacturing work;

the Quality Assurance Blueprint ( $Q A B P$ ), used to capture metrics for operations analytics, associating these with the end-to-end manufacturing processes, and

the end-to-end Process Blueprint (E2E Process BP), which ties together the many discrete processes associated with all aspects of product development, while providing the ability to adapt to changing conditions and environments.

The latter, along with the IMAGINE platform which incarnates the DMN lifecycle and the DMN management methodology [9], are used to ensure business process alignment and interoperability, and thereby information sharing, collaboration and enterprise-wide visibility, compensating thus for most of the risks potentially encountered in the DMN environment.

The dynamic and real-time nature of the BP information caters for selecting the most appropriate set of partners in the first place, as well as for efficiently coordinating the complex grid of multiple and diverse actors, functions, processes and data flows involved thereafter, ensuring thereby effective operation of the network and constant fine-tuning of its performance in case of deviations in the production plan. In addition to that, the provision of different (privilege) access rights for different partners minimizes the needless exposure of sensitive information, while the service-oriented approach adopted for the design and implementation of the IMAGINE platform makes up for the on-boarding cost involved, confining related investments to the implementation of relevant adaptors. The risk of unwanted and malicious attacks constitutes an exogenous factor which imposes the application of efficient security and authentication mechanisms, while finally, potential unethical behavior on behalf of the DMN partners, resulting in important knowledge misuse or a member's withdrawal from the network may be further controlled and diminished through the establishment of contractual agreements with special clauses on information confidentiality and the use of intellectual property, as well as specific terms legally binding the DMN members not to be able to waive their responsibilities under specific circumstances.

In this context, leveraging the CM above, it can be deduced that most of the DMN risks are inherently dealt with by the IMAGINE framework and the BP Model in specific, which in turn comes forth as the main facilitator for achieving business and IT alignment and interoperability, and thereby for supporting the creation of shared value-chain manufacturing networks.

\section{Discussion and Conclusions}

This paper presented an approach to model risk-related factors in DMNs leveraging the causal characteristics of CMs. The approach has included the elicitation of DMN 
risk-related concept variables through semi-structured interviews and their correlation through CMs. The use of CMs in this process has been observed to facilitate communication, as well as to bring forth the role of the IMAGINE framework, and interoperability in particular, in ensuring business alignment and therefore addressing the majority of DMN risks. While the derived map is quite generic to cover the majority of DMN formations and is thus open to modifications depending on the particular case, it can promote debate and further investigation. This can in turn improve managerial decision-making, and thereby the process of risk identification, assessment and mitigation.

Acknowledgments. The research leading to these results has been supported by the EC FP7 under the project "IMAGINE - Innovative end-to-end management of Dynamic Manufacturing Networks" Grant Agreement No. 285132.

\section{References}

1. Mc Clellan, M.: Manufacturing Enterprise 3.0: The New Information Management Paradigm Built On Processes. Collaboration Synergies Inc, Vancouver (2009)

2. Katzy, B.R., Schuh, G.: The Virtual Enterprise. In: Molina, A., Sánchez, J.M., Kusiak, A. (eds.) Handbook of Life Cycle Engineering: Concepts, Methods and Tools, pp. 59--92. Springer (1999)

3. IMAGINE Project: Innovative End-to-end Management of Dynamic Manufacturing Networks, www.imagine-futurefactory.eu

4. Viswanadham, N.: Partner Selection and Synchronized Planning in Dynamic Manufacturing Networks. IEEE Transactions on Robotics and Automation, 19(1), 117 - 130 (2003)

5. Papakostas, N., Efthymiou, K., Georgoulias, K., Chryssolouris, G.: On the Configuration and Planning of Dynamic Manufacturing Networks. Logistics Research Journal, 1--7 (2012)

6. Camarinha-Matos, L.M., Afsarmanesh, H., Galeano, N., Molina, A.: Collaborative networked organizations - Concepts and practice in manufacturing enterprises. Computers \& Industrial Engineering, 57(1), 46--60 (2009)

7. Hallikas, J., Karvonen, I., Pulkkinen, U., Virolainen, V.M., Tuominen, M.: Risk management processes in supplier networks. Int. J. Production Economics 90, 47--58 (2004)

8. Huff, A.S.: Mapping Strategic Thought. Wiley and Sons, USA (1990)

9. Markaki, O., Panopoulos, D., Kokkinakos, P., Koussouris, S., Askounis, D.: Towards Adopting Dynamic Manufacturing Networks for Future Manufacturing: Benefits and Risks of the IMAGINE DMN End-to-End Management Methodology. In: 22nd IEEE International Workshop on Enabling Technologies: Infrastructure for Collaborative Enterprises, pp. 305-310. IEEE Press (2013)

10. Axelrod, R.: Structure of Decision - The Cognitive Maps of Political Elites. Princeton University Press, Princeton, NJ (1976)

11. Bryson, M., Ackermann, F., Finn, C.: Visible Thinking: Unlocking causal mapping for practical business results. John Wiley \& Sons, Ltd (2004)

12. Scavarda, A.J., Chameeva, T.B., Goldstein, S.M., Hays, J.M., Hill, A.V.: Review of the Causal Mapping Practice and Research Literature. In: 2nd World Conference on POM and 15th Annual POM Conference, Cancun, Mexico (2004)

13. Martin, P.Y., Turner, B.A.: Grounded Theory and Organizational Research. The Journal of Applied Behavioral Science 22(2), 141--157 (1986) 